\section{Head tilt or head turn: neurolocalization of the head and what difference does it make?}

\section{Raquel Trevail}

In order to identify and localize intracranial lesions it is important to know the importance and function of each area and be aware of what abnormalities are expected to be found depending on the neuroanatomical localization.

Anatomically the brain can be divided into prosencephalon (telencephalon and diencephalon), mesencephalon and rhombencephalon (metencephalon and myelencephalon), but clinically we can identify forebrain, cerebellar, brainstem and vestibular syndromes.

- The forebrain includes the cerebrum and the diencephalon

- Function: the cerebral cortex is important for behaviour, vision, hearing, fine motor activity and conscious perception of touch, pain, temperature and body position. The cerebral white matter mainly conveys motor and sensory function instructions. The basal nuclei are involved in muscle tone, and initiation and control of voluntary motor activity. The diencephalon is the chief sensory integrating system of the central nervous system. It is responsible for control of autonomic and endocrine function, sleep and consciousness or awakefulness; olfactory functions; vision and pupillary light reflex; visual, auditory, nociception and proprioceptive sensory relay system to the cerebral cortex; and emotional behavioural via connections with the limbic system

- Possible clinical signs associated with forebrain disease: changes in mentation, circling to the side of the lesion, contralateral proprioceptive deficits and contralateral menace response and facial sensation deficits may be found. Seizures are a common presentation in these cases

The cerebellum plays a major role in the control of motor activity

- Function: it is a regulator and not a primary initiator of movement. It functions to coordinate and smooth out movement, including the maintenance of equilibrium and the regulation of muscle tone to preserve the normal position of the body while at rest or in motion

- Possible clinical signs: neurological signs associated with cerebellar disease vary depending on the area(s) affected but may include tremors, wide-based stance, dysmetria, absent menace response and pupil size changes and vestibular signs such as head tilt
- The brainstem includes the midbrain, pons, medulla and cerebellar peduncles. It has 10 pairs of cranial nerves (III to XII)

- Function: it contains the regulatory centres for consciousness, cardiovascular system and breathing. It links the cerebral cortex to the spinal cord through ascending and descending motor pathways

- Possible clinical signs: neurological signs usually seen with brainstem syndrome include changes in mentation and several cranial nerve abnormalities. Other findings such as cervical hyperaesthesia are possible but usually secondary to the primary problem or a diffuse pathology

- The vestibular system is the primary sensory system that maintains the animal's balance, its normal orientation relative to the gravitational field of the earth

- Function: it is responsible for maintaining the position of the eyes, neck, trunk and limbs relative to the position or movement of the head at any time. The vestibular syndrome can be divided into central and peripheral

- Possible clinical signs: the clinical signs identified with vestibular disease will depend on and reflect the localization (peripheral vs central). For example head tilt and nystagmus can be seen in both cases but proprioceptive deficits and altered mentation are consistent with a central localization.

\section{KEY LEARNING OBJECTIVES}

- Be able to localize intracranial lesions

- Relate common neurological signs to the neuroanatomical localization

- Formulate a list of differential diagnoses based on signalment and neuroanatomnical localization

\section{MUTIPLE CHOICE QUESTIONS}

1. A patient is presented with head tilt, nystagmus and no menace response. What is the neuroanatomical localization?
(A) Forebrain
(B) Brainstem
(C) Cerebellum
(D) Vestibulocochlear nerve

2. An 8-year-old Boxer presented with an history of pacing, circling to the right and lethargy. A right forebrain lesion is suspected. What other deficits might you see on the neurological examination?
(A) Proprioceptive deficits on the left thoracic and pelvic limbs
(B) Lack of menace response on the right side
(C) Proprioceptive deficits on the right thoracic and pelvic limbs
(D) Two of the above 
3. A 4-year-old Cavalier King Charles Spaniel presented with an acute onset of right head tilt and left-sided proprioceptive deficits. What is the neuroanatomical localization?
(A) Peripheral vestibular localization

(B) Central vestibular localization - brainstem

(C) Right cranial cervical localization

(D) Paradoxical vestibular localization

\section{What do you need to know about brain MRI?}

\section{Tim Trevail}

MRI is widely regarded as the imaging modality of choice in the diagnostic imaging evaluation of the neurological patient. However, an MRI scan is a very costly investigative tool and clinicians choosing this as the next appropriate test should be aware of the benefits and limitations of MRI compared with alternative imaging modalities. Benefits:

- Exquisite soft tissue detail delivers outstanding brain and spinal cord definition

- Cross-sectional imaging technique, takes away superimposition noted on radiographs

- Ability to give accurate information on tissue content such as haemorrhage or free fluid.

Limitations:

- Expensive

- Some safety considerations given the high magnetic field; staff need to be appropriately trained to avoid catastrophic incidents

- Bone imaging inferior to computed tomography for determining fractures and for surgical planning

- Relatively slow image acquisition time.

When compared to the other imaging modalities, MRI may be considered somewhat complicated. This is in part due to the complex physics associated with image acquisition but also due to the huge variety of sequences available. The most important are listed below and will be discussed during the lecture.

- T1-weighted: produced using short time to echo (TE) and time to repetition (TR) parameters and one of the staple sequences in MRI. Fluid appears dark (hypointense) and fat bright (hyperintense) on these images. T1-weighted images give good anatomical information. This sequence can be combined with the use of an intravenous gadolinium-based contrast agent, where contrast uptake indicating vascularization appears bright

- T2-weighted: produced using long TE and TR parameters and again a staple sequence. Fluid and fat both appear bright (hyperintense) on this sequence. Within the neural parenchyma, due to oedema being associated with most lesions in the brain and spinal cord, lesions are often best detected on T2-weighted images

- Fluid attenuating inversion recovery (FLAIR): this technique nulls the signal from free fluid, allowing the differentiation of free fluid (such as within a cyst or the ventricles) from oedema

- Short tau inversion recovery (STIR): this technique nulls the signal from fat and as such allows identification of oedema and reaction within paravertebral tissues, muscles and bone marrow - Gradient echo/T2*: a particularly useful sequence to identify the presence of haemorrhage or mineralization within tissues due to the presence of magnetic susceptibility effects. Gradient echo images are also good for evaluating bone

- Diffusion weighting imaging (DWI): a technique used to evaluate the diffusion of molecules (mainly water) through tissues and is very useful where cellular swelling is present (which reduces diffusion) such as in acute infarction.

Interpretation of brain MRI requires an understanding of the tissue characteristics defined by the above sequences and an understanding of the possible pathologies that may be encompassed when imaging the brain, in addition to an in-depth knowledge of brain anatomy. Several features of common brain pathologies will be discussed during this lecture.

\section{KEY LEARNING OBJECTIVES}

Understand the limitations and benefits of magnetic resonance imaging (MRI) for imaging the brain of the neurological patient

- Know what sequences are available for brain MRI, what they tell you and when to use them

- Know the interpretive principles of brain MRI and be able to identify the more common pathologies of the brain in dogs and cats

\section{MULTIPLE CHOICE QUESTIONS}

1. Which of the following features is commonly identified in extra-axial mass lesions?
(A) Poor contrast enhancement
(B) Haemorrhage
(C) Rim enhancement
(D) A dural tail 